\title{
A study of the condensed phases and solid-solid phase transition in toluene: A Monte Carlo investigation
}

\author{
A. V. Anil Kumar \\ Solid State and Structural Chemistry Unit, Indian Institute of Science, Bangalore-560012, India \\ S. Yashonatha) \\ Solid State and Structural Chemistry Unit and Supercomputer Education and Research Centre, \\ Indian Institute of Science, Bangalore-560012, India \\ S. L. Chaplot \\ Solid State Physics Division, Bhabha Atomic Research Centre, Bombay-400 085, India
}

\begin{abstract}
A Monte Carlo study of the orthorhombic $(\beta)$, monoclinic $(\alpha)$, and liquid phases of toluene in the isobaric isothermal ensemble employing variable shape simulation cell is reported here. The intermolecular potential of Williams and Starr is seen to reproduce the lattice parameters and other known properties reasonably well for the $\alpha$-phase. The $\beta$-phase is not reproduced as well. The structure has been characterized in terms of the radial distribution functions and orientational correlation functions. The transition from the orthorhombic low temperature $\beta$-phase to the high temperature monoclinic $\alpha$-phase has been successfully simulated. The transition is first order and lies between 140 and $145 \mathrm{~K}$ in agreement with experiment. The reverse transition from the $\alpha$ - to the $\beta$-phase does not take place in agreement with experiment. The liquid phase density and the heat of vaporization are reproduced well. The potential employed predicts an interaction energy which is about $5 \%$ in excess of the experimental value. The orientational correlation function and the radial distribution functions are sensitive to the potential and suggest where improvements are possible.
\end{abstract}

\section{INTRODUCTION}

Aromatic compounds such as benzene and its derivatives typically exhibit more than one solid phase. ${ }^{1}$ The detailed study of different crystalline phases and transformations between them is of considerable interest for a number of reasons. First, such a study will provide an understanding of the structure of the solid phases in terms of the nearest neighbor, next nearest neighbor, etc. arrangements. Second, a knowledge of the orientations of the neighbors with respect to each other and the degree of agreement of the simulated lattice parameters with those of the experiment would also help to obtain a fairly accurate intermolecular interaction potential. Eventually these studies are likely to provide greater understanding of the crystal structures of these systems and could lead to a more complete understanding of the problem of prediction of crystal structures of aromatic solids. Investigations carried out by Gavezotti ${ }^{2}$ and Desiraju and co-workers ${ }^{3,4}$ in this direction provide an insight into the complexity of the problem.

During the past two decades, the simulation methods have sufficiently advanced to enable their use in the study of crystal structure transformations. The method of ParrinelloRahman provides for the variation in the shape of the simulation cell and therefore can be used to study crystal structure transformations. ${ }^{5}$ The further refinement of this method and the modified implementation ${ }^{6}$ - where instead of the 9 de-

a) Also at JNCASR, Jakkur, Bangalore-560 064, India. grees of freedom for changes in the shape and size of the cell, only the necessary 6 degrees of freedom are providedhas been used by several workers to study phase transformations in a number of solids: $\mathrm{N}_{2}, \mathrm{AgI}, \mathrm{CF}_{4}, \mathrm{CCl}_{4}$, bicyclo(2.2.2)octane, adamantane, and biphenyl are some of the solids which have been investigated by this method. ${ }^{7-13}$ The transformations in all these systems are order-disorder transformations in which the ordered phase transforms into a high temperature disordered phase on heating or on change in pressure. In the case of $\mathrm{AgI}$, the $\mathrm{Ag}^{+}$sublattice melts. Further, earlier attempts to simulate the solid-solid phase transformation in benzene was not successful. ${ }^{14}$

Toluene is one of the simplest benzene derivatives which exhibits two crystalline phases- $\beta$ - and $\alpha$-toluene. The low temperature $\beta$-phase is an orthorhombic phase. The crystal structure of this phase has been determined by x-ray diffraction by Andre et al..$^{15}$ at $105 \mathrm{~K}$. The structure of high temperature $\alpha$-toluene has been investigated at $165 \mathrm{~K}$ and is monoclinic as reported by Anderson et al. ${ }^{16}$ Transformation from $\beta$-phase to $\alpha$-phase has been reported to occur between 140 and $145 \mathrm{~K}^{15}$ Laboratory experiments have found that the transformation from $\beta$ - to $\alpha$-phase is irreversible. ${ }^{15}$

In this study we shall attempt to simulate the $\beta$ - and $\alpha$-phases at $105 \mathrm{~K}$ and $165 \mathrm{~K}$, respectively. The $\beta$ - to $\alpha$-phase transformation as well as the reverse transformation have also been attempted. The intermolecular potential function of Williams and $\operatorname{Starr}^{17}$ have been employed in the present study. There have been many studies of crystal structure transformations using the variable shape constant 
pressure-constant temperature simulation. As far as we are aware all these simulations on molecular solids are of the order-disorder type of phase transition. Here we report the first study of a crystal structure transformation in a molecular solid that is not of this type. We have employed the constant pressure-constant temperature variable shape simulation cell to carry out these studies. Parrinello and Rahman did employ this method to investigate the fcc to bcc transformation in monatomic systems. ${ }^{5}$ But all studies to date on polyatomic systems have attempted simulation of the order-disorder type of transformation. Earlier investigations ${ }^{14}$ of benzene and its various condensed phases were not successful in reproducing the transformation between the orthorhombic and monoclininc phases. However this study on benzene provided two important insights: (i) The potential of Williams and $\operatorname{Cox}^{18}$ could reproduce the crystal structures reasonably well and (ii) the quadrupolar interaction is important and necessary to obtain the crystal structures correctly. Following this suggestion, we have chosen the potential of Williams and Starr $^{17}$ in the present study. This potential is derived based on a fit to the experimental crystal structure data of a number of compounds (18 in all) containing one or more benzene rings, $n$-pentane, $n$-hexane, $n$-octane, cubane, adamantane, etc. The presence of electrostatic interactions between neighboring molecules through site-site interactions account for the quadrupole moment. The liquid phase of toluene has also been investigated.

We also calculate the lattice dynamics in the two crystalline solid phases of toluene and the consequent changes in the free energy ${ }^{19}$ within the quasiharmonic approximation. The quasiharmonic results predict the stability of the $\beta$-phase at all temperatures upto melting whereas the $\beta$ - to $\alpha$-phase transformation is observed in the fully anharmonic MC simulations. This comparison suggests that the phase transformation is driven by anharmonicity of the lattice vibrations.

\section{INTERMOLECULAR POTENTIAL AND COMPUTATIONAL DETAILS}

Parameter set II of Williams and Starr ${ }^{17}$ has been used in this study. There are 15 sites per toluene molecule, one on each of the carbon and hydrogen atoms. The site-site interaction is of the 6-exp form with a Coulomb term,

$$
\Phi_{i j}=-A_{i j} / r_{i j}^{6}+B_{i j} \exp \left(-C_{i j} r_{i j}\right)+q_{i} q_{j} / r_{i j} .
$$

The net charge on $\mathrm{CH}$ as well as $\mathrm{CH}_{3}$ is zero. In other words,

$$
q_{\mathrm{H}}+q_{\mathrm{C}}=0
$$

for the $\mathrm{CH}$ group and

$$
3 q_{\mathrm{H}}^{\prime}+q_{\mathrm{C}}^{\prime}=0
$$

for the $\mathrm{CH}_{3}$ group where $q_{\mathrm{H}}, q_{\mathrm{C}}, q_{\mathrm{H}}^{\prime}$, and $q_{\mathrm{C}}^{\prime}$ are, respectively, the charges of hydrogen and carbon of the $\mathrm{CH}$ and the $\mathrm{CH}_{3}$ groups, respectively. Table I lists the potential parameters.

The total intermolecular energy is given by

$$
U=\frac{1}{2} \sum_{k=1}^{N} \sum_{l=1}^{N} \sum_{i=1}^{15} \sum_{j=1}^{15} \Phi_{i j}^{k l} \text {. }
$$

TABLE I. The potential parameters of Williams and Starr (Ref. 17) used in the simulation.

\begin{tabular}{lc}
\hline \hline Parameter & Value \\
\hline$A_{\mathrm{HH}}$ & $136 \mathrm{~kJ} \AA^{6} / \mathrm{mol}$ \\
$B_{\mathrm{HH}}$ & $11677 \mathrm{~kJ} / \mathrm{mol}$ \\
$C_{\mathrm{HH}}$ & $3.74 \AA^{-1}$ \\
$A_{\mathrm{CH}}$ & $573 \mathrm{~kJ} \AA^{6} / \mathrm{mol}$ \\
$B_{\mathrm{CH}}$ & $65485 \mathrm{~kJ} / \mathrm{mol}$ \\
$C_{\mathrm{CH}}$ & $3.67 \AA^{-1}$ \\
$A_{\mathrm{CC}}$ & $2414 \mathrm{~kJ} \AA^{6} / \mathrm{mol}$ \\
$B_{\mathrm{CC}}$ & $367250 \mathrm{~kJ} / \mathrm{mol}^{-1}$ \\
$C_{\mathrm{CH}}$ & $3.60 \AA^{-1}$ \\
$q_{\mathrm{H}}(\mathrm{CH})$ & $+0.153|e|$ \\
$q_{\mathrm{C}}(\mathrm{CH})$ & $-0.153|e|$ \\
$q_{\mathrm{H}}(\mathrm{CH})$ & $+0.0765|e|$ \\
$q_{\mathrm{C}}\left(\mathrm{CH} \mathrm{H}_{3}\right)$ & $-0.2295|e|$ \\
\hline \hline
\end{tabular}

All simulations were carried out in the constant pressureconstant temperature (NPT) ensemble using the well known importance sampling technique of Metropolis et al. ${ }^{20}$ Following Yashonath and Rao, ${ }^{6}$ one, two, and three degrees of freedom are provided for vectors $\mathbf{a}, \mathbf{b}$, and c, respectively. This provides the necessary six degrees of freedom for the simulation cell to be in any one of the seven crystal systems. Each Monte Carlo step consists of an attempted displacement plus rotation along a randomly chosen axis for each of the $N$ molecules and displacement of the simulation cell vectors $\mathbf{a}, \mathbf{b}$, and $\mathbf{c}$. Each of the condensed phases $(\beta-, \alpha-$, and liquid) were equilibrated over $1000 \mathrm{MC}$ steps and properties averaged over $2000 \mathrm{MC}$ steps. The $\beta$ - to $\alpha$-transition was carried out over $6000 \mathrm{MC}$ steps. A c.o.m.-c.o.m. cutoff of $12 \AA$ was employed. The estimated error in the Coulomb term is less than $1 \%$ and in the total energy the error is less than $2 \%$. No neighbor list was employed to compute interactions. All simulations were carried at a constant pressure of 1 atm.

For the $\beta$-phase, a total of 128 molecules $(2 \times 2 \times 4$ crystallographic unit cells) have been used. Note that there are 8 molecules in one crystallographic unit cell $(Z=8)$ for both $\beta$ - and $\alpha$-phases. Table II lists the crystal structure parameters of the $\beta$ - and $\alpha$-phases of toluene as reported from $\mathrm{x}$-ray diffraction measurements. For the $\alpha$-phase $4 \times 4 \times 1$ unit cells consisting of 128 molecules were used. $\beta$ - to $\alpha$-phase transformation was attempted on a $2 \times 2 \times 4$ system of the $\beta$-phase while the liquid phase was obtained by heating the final configuration of the $\alpha$-phase $(4 \times 4 \times 1$ unit cells).

Altogether six runs were carried out: I, Ia, II, IIa, III, IIIa, and IV. Run I and Ia relate to the simulation of the $\beta$-phase at $105 \mathrm{~K}$ in the NPT and NVT ensemble, respectively. Similarly run II and IIa relate to the simulation of $\alpha$-phase at $165 \mathrm{~K}$ in the NPT and NVT ensemble. The NVT runs were carried out with $\mathbf{a}, \mathbf{b}$, and $\mathbf{c}$ simulation cell vectors given by the x-ray measurements. There was no change in volume or the cell vectors. Further, Ia and IIa were carried out only for a short duration of $100 \mathrm{MC}$ steps at the appropriate temperature to thermalize the experimental structure. Initial configuration was the reported experimental $\mathrm{x}$-ray structure. Periodic boundary conditions are employed in all 
TABLE II. Unit cell parameters of the $\beta$-phase and $\alpha$-phase of toluene from x-ray crystallographic data and variable shape Monte Carlo simulation.

\begin{tabular}{|c|c|c|c|c|c|}
\hline & \multicolumn{2}{|c|}{$\beta$-phase $(105 \mathrm{~K})$} & \multicolumn{2}{|c|}{$\alpha$-phase (165 K) } & \multirow{2}{*}{$\begin{array}{c}\alpha \text {-phase }(165 \mathrm{~K}) \\
(\beta \rightarrow \alpha \text {-phase })\end{array}$} \\
\hline & VS MC & X-ray $(\text { Expt. })^{\mathrm{a}}$ & VS MC & $\mathrm{X}$-ray $\left(\right.$ Expt.) ${ }^{\mathrm{b}}$ & \\
\hline $\mathbf{a}(\AA)$ & $14.06(1.5)$ & 13.85 & $7.69(0.3)$ & 7.67 & 7.81 \\
\hline $\mathbf{b}(\AA)$ & $10.91(4.7)$ & 11.42 & $5.77(1.0)$ & 5.83 & 5.68 \\
\hline $\mathbf{c}(\AA)$ & $7.58(2.5)$ & 7.39 & $26.75(0.9)$ & 26.98 & 27.13 \\
\hline$\alpha(\operatorname{deg})$ & 89.96 & 90.0 & 89.77 & 90.0 & 89.97 \\
\hline$\beta(\operatorname{deg})$ & 89.77 & 90.0 & $105.25(0.45)$ & 105.73 & 103.92 \\
\hline$\gamma(\operatorname{deg})$ & 89.97 & 90.0 & 90.31 & 90.0 & 89.98 \\
\hline $\begin{array}{l}\mathrm{V}\left(\mathrm{A}^{3} /\right. \\
\text { molecule })\end{array}$ & 145.4 & 146.1 & 146.1 & 145.1 & 146.07 \\
\hline$Z$ & 8 & 8 & 8 & 8 & 8 \\
\hline $\begin{array}{l}\mathrm{U}(\mathrm{kJ} / \mathrm{mol}) \\
\text { space group }\end{array}$ & -52.3 & $\begin{array}{c}\cdots \\
P b c n\end{array}$ & -50.4 & $\begin{array}{c}\cdots \\
P 2{ }_{1} c\end{array}$ & -49.8 \\
\hline
\end{tabular}

${ }^{\mathrm{a}}$ From Ref. 15.

${ }^{\mathrm{b}}$ From Ref. 16.

the three directions. Run III attempts to simulate the $\beta$ - to $\alpha$-phase transformation in the NPT ensemble while run IV is an NPT ensemble simulation of liquid toluene. Run IIIa is an independent run in which the starting configuration was the final configuration of run I. The temperature was raised in steps of $5 \mathrm{~K}$ starting from $105 \mathrm{~K}$ until a temperature of 165 $\mathrm{K}$ was reached. All NPT ensemble simulations were carried out along with a variation in $\mathbf{a}, \mathbf{b}$, and $\mathbf{c}$ vectors.

\section{RESULTS AND DISCUSSION}

To begin with our aim is to see how well the potential of Williams and $\operatorname{Starr}^{17}$ can reproduce the $\beta$ - and $\alpha$-phases of toluene.

\section{A. Structure of $\beta$-toluene at $105 \mathrm{~K}$}

The starting configuration for run I and Ia was taken to be the crystal structure reported by Andre et al. ${ }^{15}$ The simulation was carried out on $2 \times 2 \times 4$ unit cells consisting of 128 molecules of toluene. The result of this run are listed in Table II. It is seen that the lattice parameters $a, b, c$ and $\alpha, \beta$, $\gamma$ are within $5 \%$ of the values reported by Andre et al. ${ }^{15}$ The largest deviation (listed within parentheses) is found to be that of the $b$ parameter. The three angles remain close to $90^{\circ}$ suggesting that the potential of Williams and Starr predicts a stable orthorhombic phase. The volume of the unit cell is within $1 \%$ of the experimentally reported value.

The structure of this solid phase can be understood in terms of the radial distribution functions (rdfs). There are four site-site radial distribution functions that can be defined, viz., center-of-mass-center-of-mass (c.o.m.-c.o.m.), $\mathrm{C}_{\text {ring }}-\mathrm{C}_{\text {ring }}, \mathrm{C}_{\text {ring }}-\mathrm{C}_{\mathrm{CH}_{3}}$ and $\mathrm{C}_{\mathrm{CH}_{3}}-\mathrm{C}_{\mathrm{CH}_{3}}$. We have not tried to distinguish between different ring carbon positions in computing $\mathrm{C}_{\text {ring }}-\mathrm{C}_{\text {ring }}$. Figure 1 shows the four radial distribution functions for the $\beta$-phase. The constant volume simulation (run Ia) corresponds to the structure close to the actual crystalline phase since the lattice parameters are not allowed to change and the run is short. The continuous line (run I) which shows the c.o.m.-c.o.m. $\mathrm{g}(r)$ [see Fig. 1(a)] for the variable shape constant pressure simulation is slightly different from the experimental (run Ia) $\mathrm{g}(r)$ (dotted-dashed line).
The single peak in the run Ia near $4.7 \AA$ has split into two peaks. The $6 \AA$ peak is shifted towards a lower value of $r$. It is seen that the c.o.m.-c.o.m. $\mathrm{g}(r)$ starts at around $4.3 \AA$ in the experimental $\mathrm{g}(r)$ (obtained from run Ia) as compared to around $4.1 \AA$ in the variable shape constant pressure simulation. This shift is consistent with the slightly lower value $(0.5 \%)$ for the volume of the unit cell obtained from VS-MC (run I) simulation. The presence of diffuse peaks in the $\mathrm{C}_{\text {ring }}-\mathrm{C}_{\text {ring }}$ and $\mathrm{C}_{\text {ring }}-\mathrm{C}_{\mathrm{CH}_{3}} \mathrm{~g}(r)$ shown in Fig. 1(c) is expected since there are 6 ring carbons. The three predominent

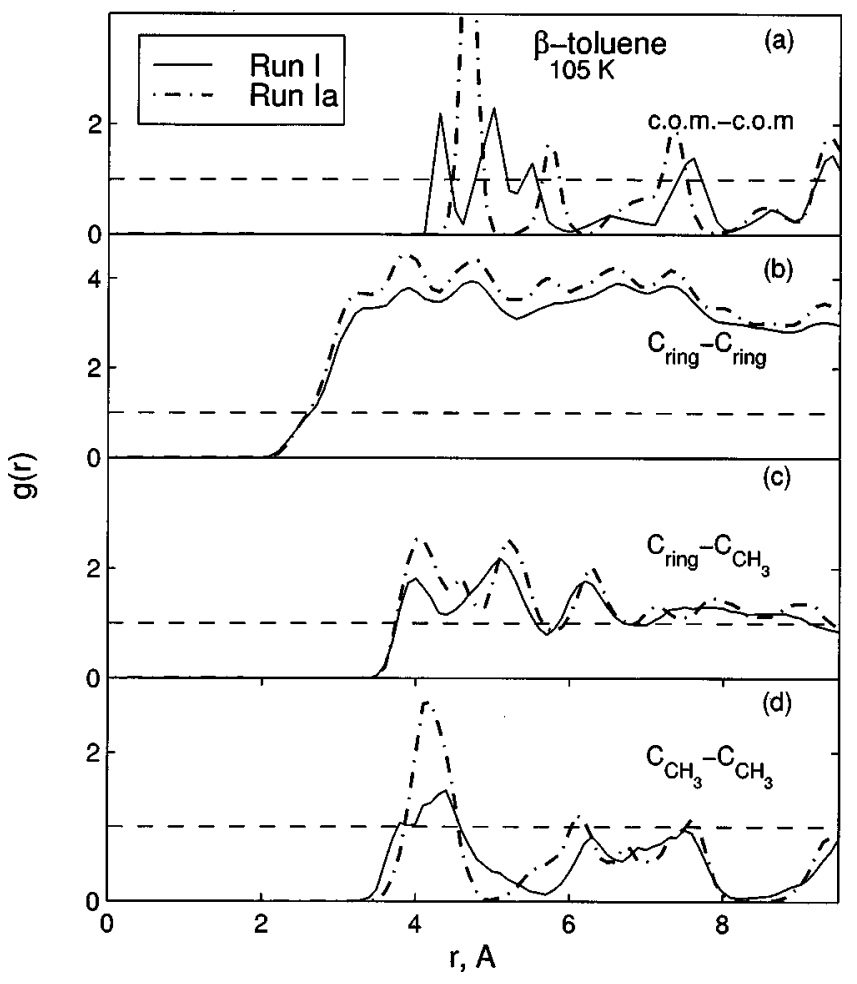

FIG. 1. The c.o.m.-c.o.m., $\mathrm{C}_{\text {ring }}-\mathrm{C}_{\text {ring, }} \mathrm{C}_{\text {ring }}-\mathrm{C}_{\mathrm{CH}_{3}}$, and $\mathrm{C}_{\mathrm{CH}_{3}}-\mathrm{C}_{\mathrm{CH}_{3}}$ radial distribution functions of the $\beta$-phase of toluene at $105 \mathrm{~K}$. The solid line corresponds to the $\mathrm{g}(r)$ 's obtained from the variable shape simulation (run I) where as the dotted-dashed line have been obtained from the NVT MC run (run Ia). 


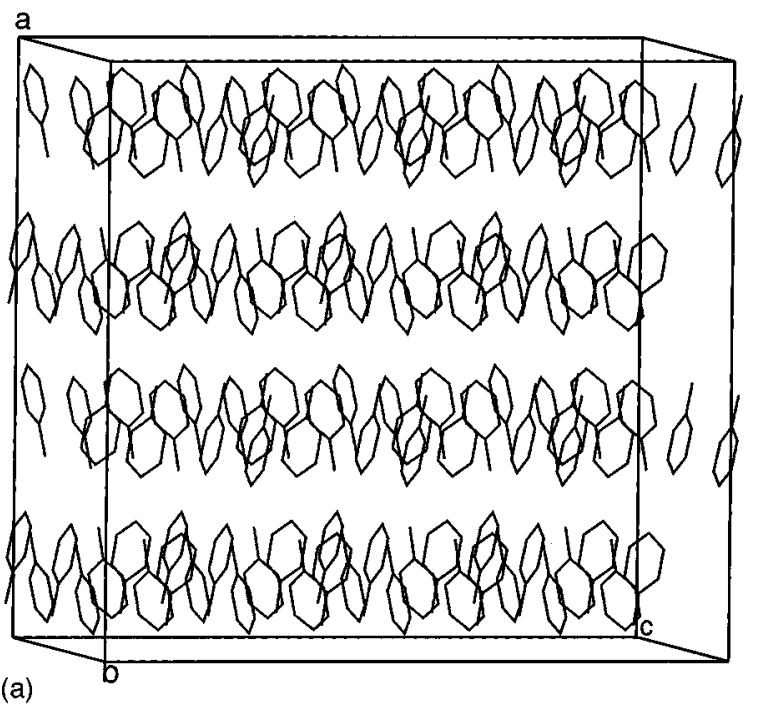

(a)

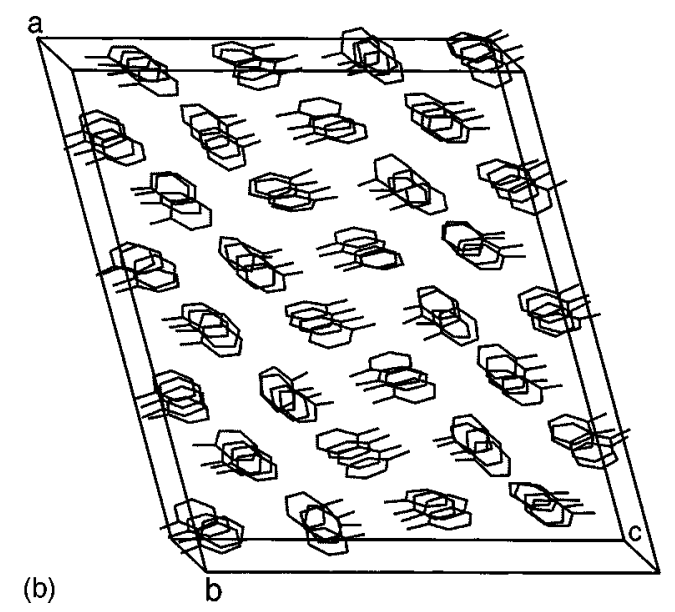

FIG. 2. A snapshot of (a) $\beta$-phase at $105 \mathrm{~K}$ and (b) $\alpha$-phase at $165 \mathrm{~K}$ obtained from the MC simulation.

peaks seen in the $\mathrm{C}_{\mathrm{CH}_{3}}-\mathrm{C}_{\mathrm{CH}_{3}} \mathrm{~g}(r)$ obtained from simulation [Fig. 1(d)] may be compared to the fine structure observed in the experimental curve. This suggests that there is reasonable librational and vibrational motion of the $\mathrm{CH}_{3}$ group. An instantaneous snapshot of the simulated $\beta$-toluene phase is shown in Fig. 2(a). The intensities of the peaks obtained from run I and Ia are not expected to agree with each other since run Ia has been carried out for a very limited number of MC steps without proper equilibration just to obtain the peak positions corresponding to the experimentally reported crystal structure. Figure 2(b) will be discussed later in the next section.

An insight into orientational correlations among toluene in the $\beta$-phase can be obtained from two correlation functions defined $\operatorname{as}^{21,14}$

$$
\begin{aligned}
& \phi_{202}(R)=(1 / 4 \sqrt{5})\left\langle 3\left(\hat{\mathbf{z}}_{1} \cdot \hat{\mathbf{R}}\right)^{2}+3\left(\hat{\mathbf{z}}_{2} \cdot \hat{\mathbf{R}}\right)^{2}-2\right\rangle, \\
& \phi_{220}(R)=(1 / 2 \sqrt{5})\left\langle 3\left(\hat{\mathbf{z}}_{1} \cdot \hat{\mathbf{z}}_{2}\right)^{2}-1\right\rangle .
\end{aligned}
$$

These two functions together indicate the orientation of two molecules with respect to each other at various distances of separation $R$. Here $\hat{R}$ is the unit vector connecting the c.o.m. of two neighboring molecules (1 and 2) and $\hat{z}_{1}$ and $\hat{z}_{2}$

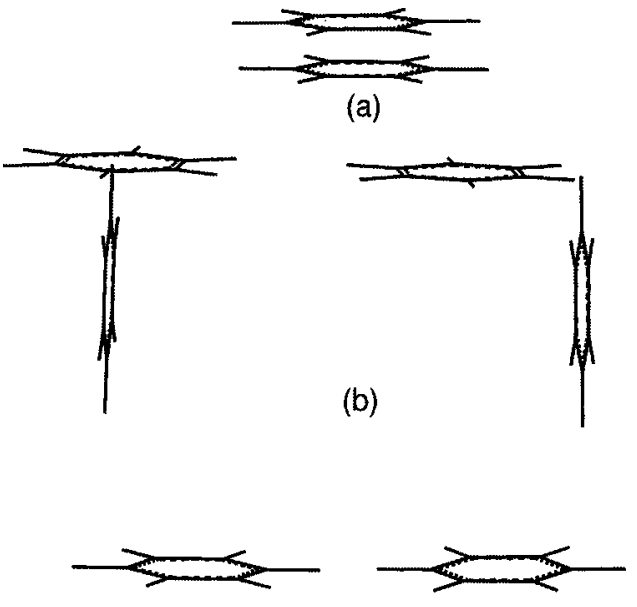

(c)
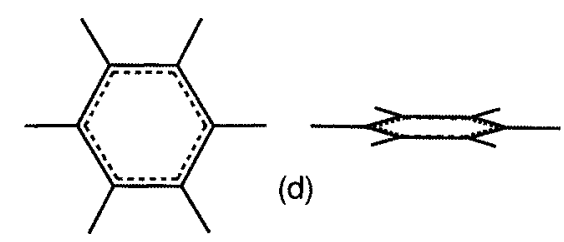

(d)

FIG. 3. The orientational correlation functions $\phi_{202}(r)$ and $\phi_{220}(r)$ together indicate the orientation of two molecules with respect to each other. (a) Stacked configuration for which $\phi_{202}(r)$ and $\phi_{220}(r)$ are both positive. (b) $\mathrm{T}$ - and L-shaped configuration for which $\phi_{202}(r)>0$ and $\phi_{220}(r)<0$. (c) Side by side configuration in which the two rings line in a single plane beside each other. Here $\phi_{202}(r)<0$ and $\phi_{220}(r)>0$. (d) Out-of-plane configuration for which both $\phi_{202}(r)$ and $\phi_{220}(r)$ are negative.

are the vectors perpendicular to the plane of the benzene ring of molecule 1 and 2, respectively. Figure 3 depicts the various possibilities. For a stacked configuration in which the two benzenes lie parallel to each other, $\phi_{202}(R)$ and $\phi_{220}(R)$ are both positive [see Fig. 3(a)]. In a T-shaped or L-shaped arrangement of benzene rings, note that $\phi_{202}(R)>0$ and $\phi_{220}(R)<0$ [see Fig. 3(b)]. $\phi_{202}(R)<0$ and $\phi_{220}(R)>0$ suggests that the two rings lie in a single plane beside each other. These are shown in Fig. 3(c). When both $\phi_{202}(R)$ and $\phi_{220}(R)$ are negative, the orientation is as shown in Fig. $3(d)$. Figure 4 shows these correlation functions for the crys-

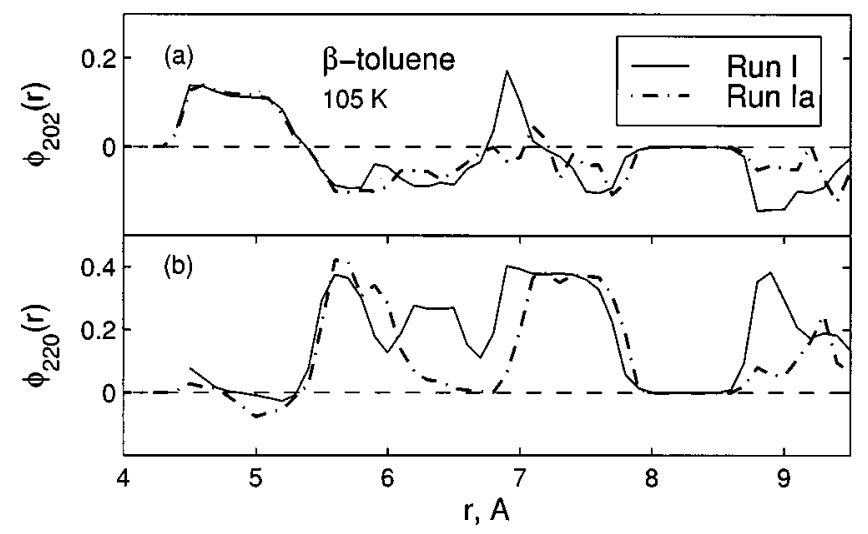

FIG. 4. The orientational correlation functions $\phi_{202}(r)$ and $\phi_{220}(r)$ of $\beta$-toluene at $105 \mathrm{~K}$. The details are same as in Fig. 1. 
talline $\beta$-phase from runs I(continuous line) and Ia (dotteddashed line). It is seen that the interaction potential predicts the correct structure for the equilibrated $\beta$-phase. There is reasonable agreement with the experimental crystal structure. There are some differences in $\phi_{220}(r)$ in the range: $6.0-7.3$ $\AA$ and $8.6-9.1 \AA$. But note that these differences are really not significant since the number of neighbors at these distances (see Fig. 1) are negligible. The nearest neighbor structure is characterized by $\phi_{202}(r)>0$ and $\phi_{220}(r)<0$ (between 4.7 and $5.3 \AA$ ) suggesting a T-shaped arrangement. Earlier studies of the crystalline phases of the benzene as well as simulations of the solid and liquid phases of benzene has suggested that the most favored nearest neighbor arrangement is a T-shaped configuration. ${ }^{14}$ It is also known that the T-shaped configuration is lower in energy than the stacked configuration (in which the two rings are stacked over one another parallel to each other) due to the quadrupole interactions.

Between 5.5 and $6.0 \AA, \phi_{202}(r)<0$ and $\phi_{220}(r)>0$ suggesting that the two benzene rings lie in the same plane beside each other with rings embedded in the plane. Between 6.8 and $8.0 \AA$, both $\phi_{202}(r)$ and $\phi_{220}(r)$ are positive suggesting a stacked (parallel) configuration. The quadrupolar interactions which vary as $r^{-5}$ do not play a significant role at these large distances. Note that Fig. 4 should be interpreted in conjunction with Fig. 1 since the density of population at a given distance can be obtained only from the latter.

\section{B. Structure of $\alpha$-toluene at $165 \mathrm{~K}$}

The unit cell parameters obtained from variable shape Monte Carlo simulation (run II) are listed in Table II. The initial configuration was taken to be the $\mathrm{x}$-ray structure of the $\alpha$-phase reported by Anderson et al. ${ }^{16}$ It is seen that the angle $\beta$ remains close to the experimentally obtained value while other two angles are close to $90^{\circ}$. The unit cell lengths are within $1 \%$ of the experimental values. The volume is again found to be close to the experimental volume.

Four different radial distribution functions (rdfs) for the $\alpha$-phase are shown in Fig. 5. In general the $\mathrm{g}(r)$ 's for the $\alpha$-phase are more diffuse than the $\mathrm{g}(r)$ 's of $\beta$-phase as is to be expected. A snapshot of the $\alpha$-phase at $165 \mathrm{~K}$ is shown in Fig. 2(b). It is evident that the ordering of the toluene is different from those found in the $\beta$-phase. The simulated rdfs show much better agreement with the experimental or NVT MC rdfs.

Figure 6 shows a plot of the $\phi_{202}(r)$ and $\phi_{220}(r)$ for the $\alpha$-phase. It is seen that these functions for the $\alpha$-phase are quite different from those obtained for the low temperature $\beta$-phase. The nearest neighbor configuration below a distance of $5 \AA$ is similar to that observed for the $\beta$-phase, viz., $\phi_{202}(r)>0$ and $\phi_{220}(r)<0$ suggesting a T-shaped orientation with respect to each other. $\phi_{220}(r)$ shows rather poor agreement in the vicinity of $6 \AA$ indicating thereby that the rings are not in the experimentally derived orientation. Note that this is in spite of the fact that the rdfs show excellent agreement (see Fig. 5). But this is because there could be several orientations which could all give the same rdfs. This is especially true since $\mathrm{C}_{\text {ring }}-\mathrm{C}_{\text {ring }}$ do not distinguish between various carbons of the ring.

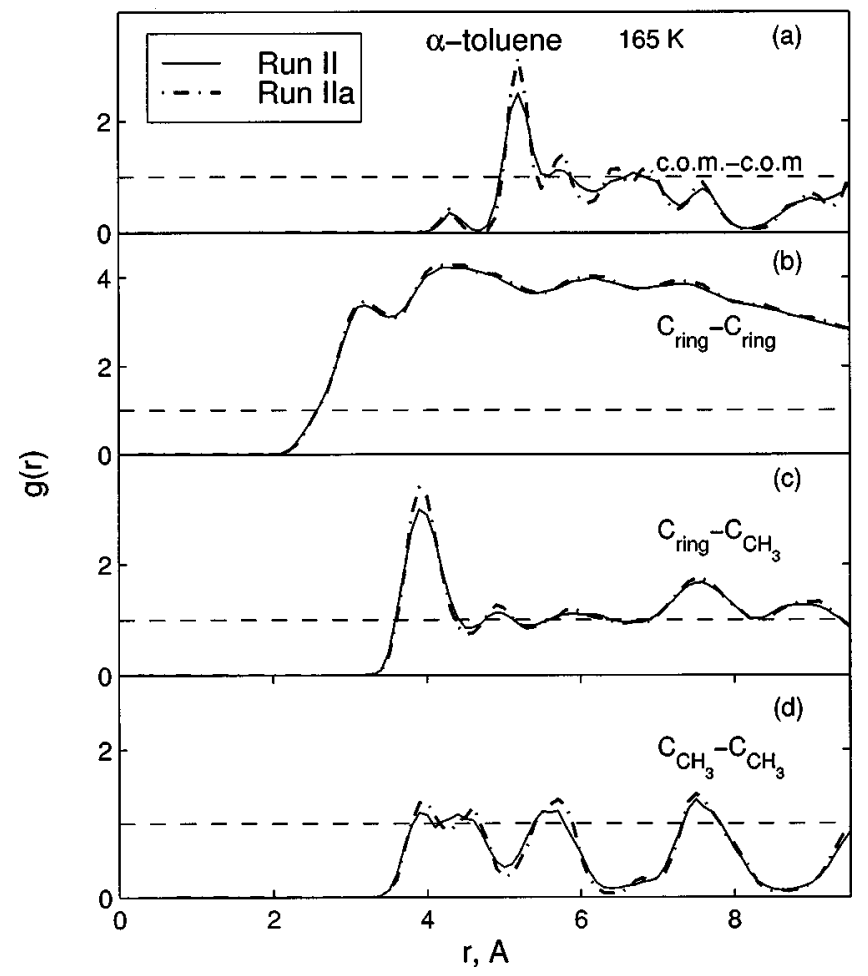

FIG. 5. The c.o.m.-c.o.m., $\mathrm{C}_{\text {ring }}-\mathrm{C}_{\text {ring }}, \mathrm{C}_{\text {ring }}-\mathrm{C}_{\mathrm{CH}_{3}}$, and $\mathrm{C}_{\mathrm{CH}_{3}}-\mathrm{C}_{\mathrm{CH}_{3}}$ radial distribution functions of the $\alpha$-phase of toluene at $165 \mathrm{~K}$. The solid lines are the $\mathrm{g}(r)$ 's obtained from the variable shape simulation (run II). The dotteddashed lines show those calculated from NVT MC run (run IIa).

Just beyond $7 \AA$ it is seen that $\phi_{202}(r)$ and $\phi_{220}(r)$ are both positive over a narrow range suggesting a stacked arrangement. Such an arrangement is seen around $8.3 \AA$ as well. Stacked configuration is also seen over a narrow range between 5.2 and $5.5 \AA$. This suggests that in case of toluene the stacked configuration is destabilized only at the distance of closest approach $(<5 \AA)$. In benzene, the role of the quadrupole moment is predominant. Presumably, the $\mathrm{CH}_{3}$ group in toluene interacts favorably with the ring. This seems to be responsible for the decreased role of the quadrupolar interactions in determining the orientation of the rings between neighbors beyond $5.5 \AA$ distance.

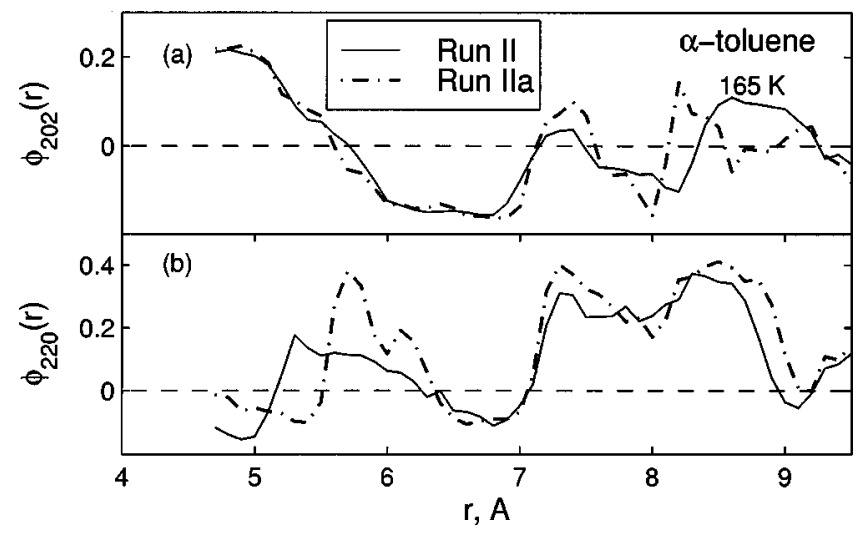

FIG. 6. The orientational correlation functions $\phi_{202}(r)$ and $\phi_{220}(r)$ of $\alpha$-toluene at $165 \mathrm{~K}$. The details are same as in Fig. 5. 


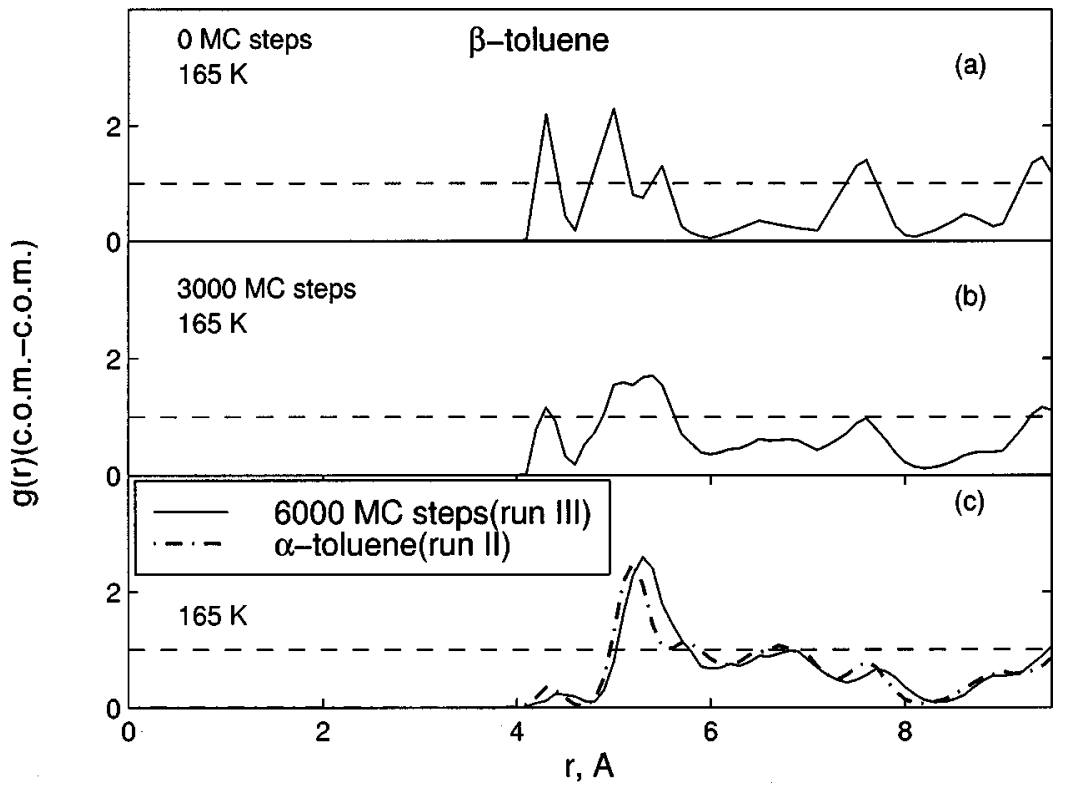

FIG. 7. The change in the c.o.m.-c.o.m. $\mathrm{g}(r)$ during the $\beta$ - to $\alpha$-phase transition of toluene. (a) The c.o.m.c.o.m. $\mathrm{g}(r)$ of the starting configuration. (b) After 3000 MC steps. (c) After $6000 \mathrm{MC}$ steps (continuous line). The $\mathrm{g}(r)$ obtained from the run II of $\alpha$-toluene is shown as a dotted-dashed line for comparison.

\section{C. $\beta$ - to $\alpha$-phase structural transformation}

Starting with the $\beta$-phase structure and a temperature of $105 \mathrm{~K}$, long runs with a variable shape simulation cell at constant pressure (run III) was carried out. The temperature was increased to $165 \mathrm{~K}$ and the c.o.m.-c.o.m. $\mathrm{g}(r)$ was monitored to see if there was any change in the solid phase. Figure 7 displays these rdfs at the start and after 3000 and $6000 \mathrm{MC}$ steps. It is evident from the $\mathrm{g}(r)$ at $3000 \mathrm{MC}$ steps that the structure is undergoing a transformation. During the first 4000 or $5000 \mathrm{MC}$ steps, the instantaneous $\mathrm{g}(r)$ calculated at any given MC step was seen to be evolving. Beyond 5000 MC steps, the $\mathrm{g}(r)$ remained the same and did not show any further evolution or change with the MC steps. The $\mathrm{g}(r)$ after $6000 \mathrm{MC}$ steps is plotted in Fig. 7(c). The c.o.m.c.o.m. $\mathrm{g}(r)$ of Fig. 3 for the $\alpha$-phase(run II) is also shown in the Fig. 7(c). It is evident that both have similar structure, namely, that of the monoclinic $\alpha$-phase. Figure 8 shows a plot of $\mathrm{C}_{\text {ring }}-\mathrm{C}_{\mathrm{CH}_{3}} \mathrm{~g}(r)$ at the start of simulation, after 3000 and $6000 \mathrm{MC}$ steps. It is clear that the $\mathrm{g}(r)$ after $6000 \mathrm{MC}$ steps is close to that of the $\alpha$-phase(indicated by the dotteddashed line). Figures 9 and 10 show the evolution of orientational correlation functions $\phi_{202}(r)$ and $\phi_{220}(r)$ over 6000 MC steps. These closely overlap those obtained from run III. Thus, clearly the low temperature orthorhombic $\beta$-phase has transformed into the monoclinic $\alpha$-phase, at $165 \mathrm{~K}$. The simulation cell parameters of the transformed phase for run III as well as for run I and II are listed in Table II. It is seen that the lattice parameters of the $\alpha$-phase from run III and II agree closely with each other. The relationship between the unit cell parameters of the $\alpha$ - and the $\beta$-phases is as follows:

$$
\begin{aligned}
& a_{\alpha}=c_{\beta}, \\
& b_{\alpha}=b_{\beta} / 2,
\end{aligned}
$$

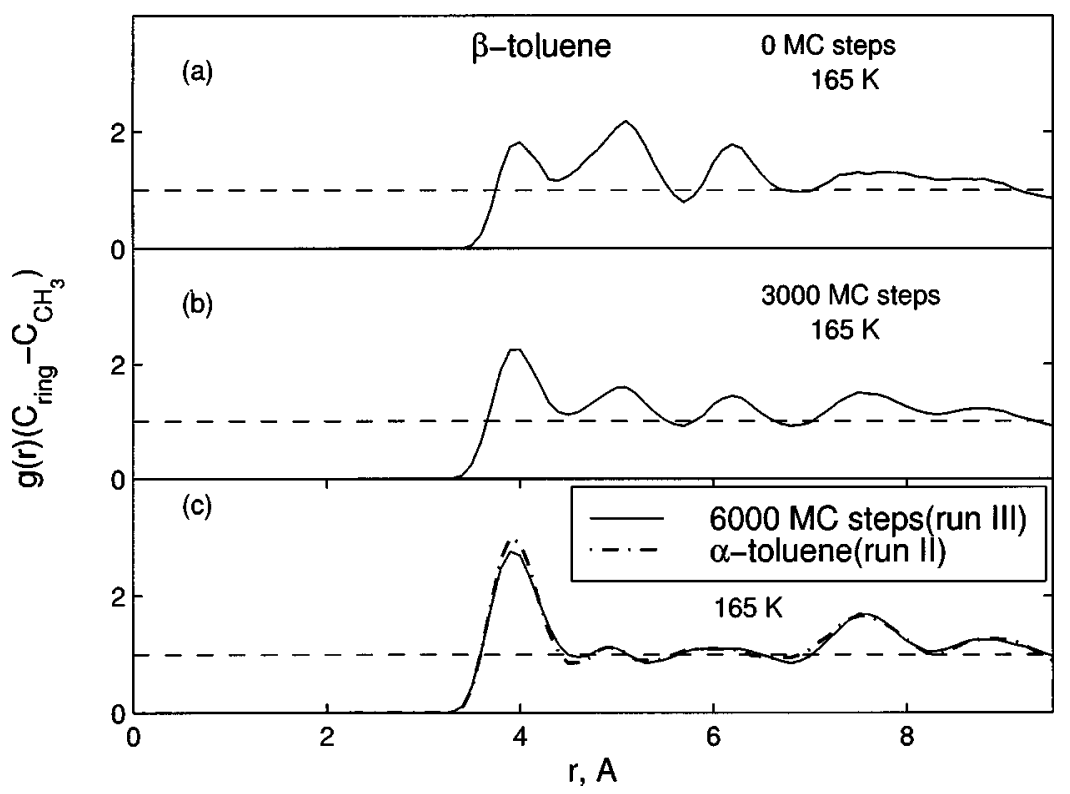

FIG. 8. The evolution of the $\mathrm{C}_{\text {ring }}-\mathrm{C}_{\mathrm{CH}_{3}} \mathrm{~g}(r)$ during the $\beta$ - to $\alpha$-phase transition of toluene. (a) The $\mathrm{C}_{\text {ring }}-\mathrm{C}_{\mathrm{CH}_{3}}$ $\mathrm{g}(r)$ of the starting configuration. (b) After $3000 \mathrm{MC}$ steps. (c) After $6000 \mathrm{MC}$ steps(continuous line). The $\mathrm{g}(r)$ obtained from the VS-MC run (run II) of $\alpha$-toluene is shown (dotted-dashed line) for comparison. 


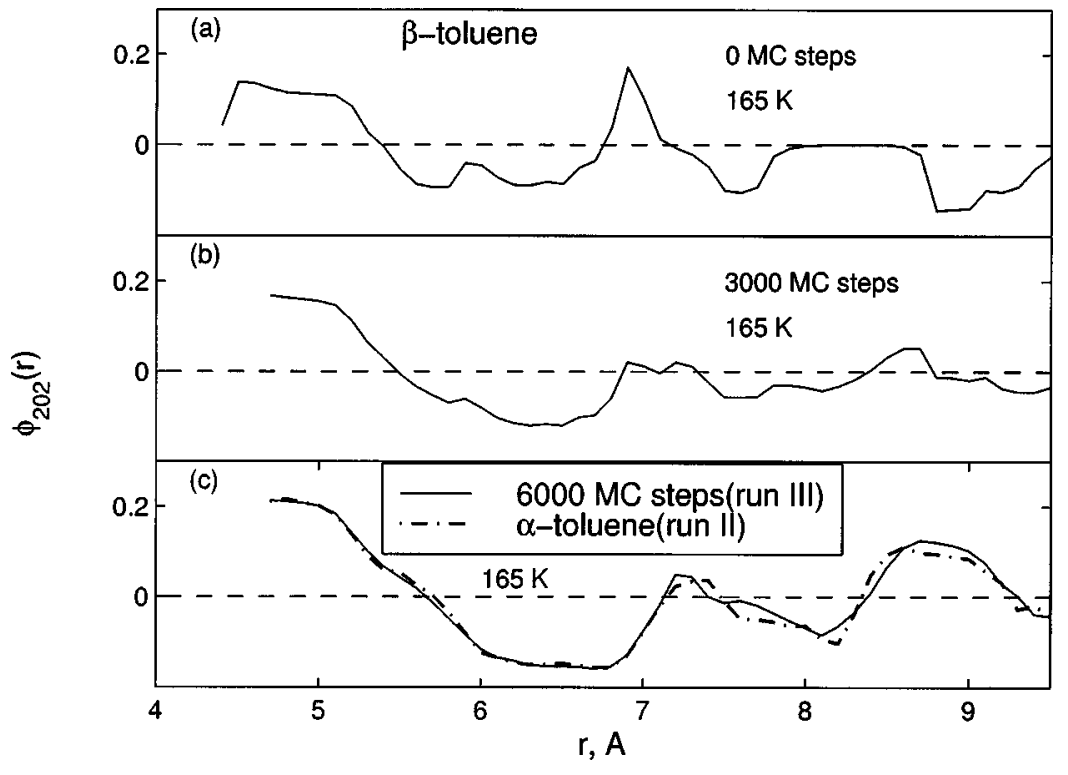

FIG. 9. The change in the orientation correlation function $\phi_{202}(r)$ during the $\beta$ - to $\alpha$-phase transition of toluene. (a) The $\phi_{202}(r)$ of the starting configuration. (b) After 3000 MC steps. (c) After 6000 MC steps. The dotted-dashed line shows the orientational correlation function $\phi_{202}(r)$ obtained from the VS-MC run of $\alpha$-toluene (run II).

$$
c_{\alpha}=2 \alpha_{\beta} \text {. }
$$

There is little change in the volume of the unit cells when the structure transforms from $\beta$ - to the $\alpha$-phase. The most significant change occurs in the angle $\beta$ which changes from $90^{\circ}$ to $103.9^{\circ}$.

We attempted to characterize the $\beta$ - to $\alpha$-transformation more carefully by carrying out yet another simulation, run IIIa. Here we started with the $\beta$-phase at $105 \mathrm{~K}$ as in run III but raised the temperature in steps of $5 \mathrm{~K}$ until we reached $165 \mathrm{~K}$. At each temperature, the system was equilibrated for 1000 MC steps and averages obtained over next $2000 \mathrm{MC}$ steps. The starting configuration at any given temperature was taken from the final configuration of the previous temperature. The evolution of average intermolecular energy $\langle U\rangle$ and lattice parameters $a, b, c$, and $\beta$ are shown in Fig. 11. It is seen that up to $140 \mathrm{~K}$ there is little or no change in the lattice parameters and $U$. The change in volume is small and hence is not plotted. Transition temperature clearly lies between 140 and $145 \mathrm{~K}$. This is in excellent agreement with the experimental range of $140-145 \mathrm{~K}$ for the transition temperature. The latter is based on X-ray diffraction data of Andre et al. ${ }^{15}$ Figure 11 suggests that though the volume change is small, the $\beta$ - to $\alpha$-phase transformation is a firstorder transition.

The fact that a change of this magnitude does occur in the simulation by a raise of just $5^{\circ}$ (as we will see below) in the temperature augurs well for the Parrinello-Rahman variable shape simulation method. Earlier when simulations were attempted on monatomic solids, the transition from fcc to hep did not take place. ${ }^{5,22}$ In a fcc to hep transition, modification near the third or the fourth shell of neighbors is necessary. In contrast, in toluene a modification of the first two shells-nearest neighbor and next nearest neighboritself occurs as is indicated by the c.o.m.-c.o.m. $\mathrm{g}(r)$ (see Fig. 7).

To the best of our knowledge this is the first time that a

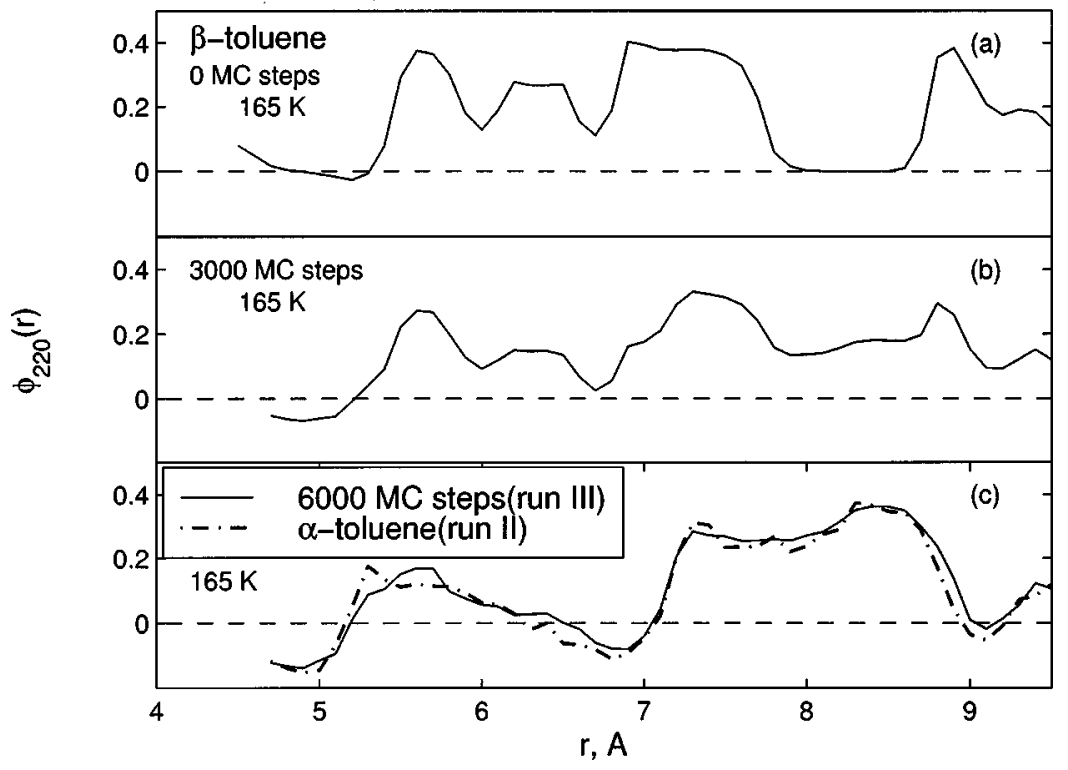

FIG. 10. The change in the orientation correlation function $\phi_{202}(r)$ during the $\beta$ - to $\alpha$-structural phase transition of toluene. Details are as in Fig. 9. 


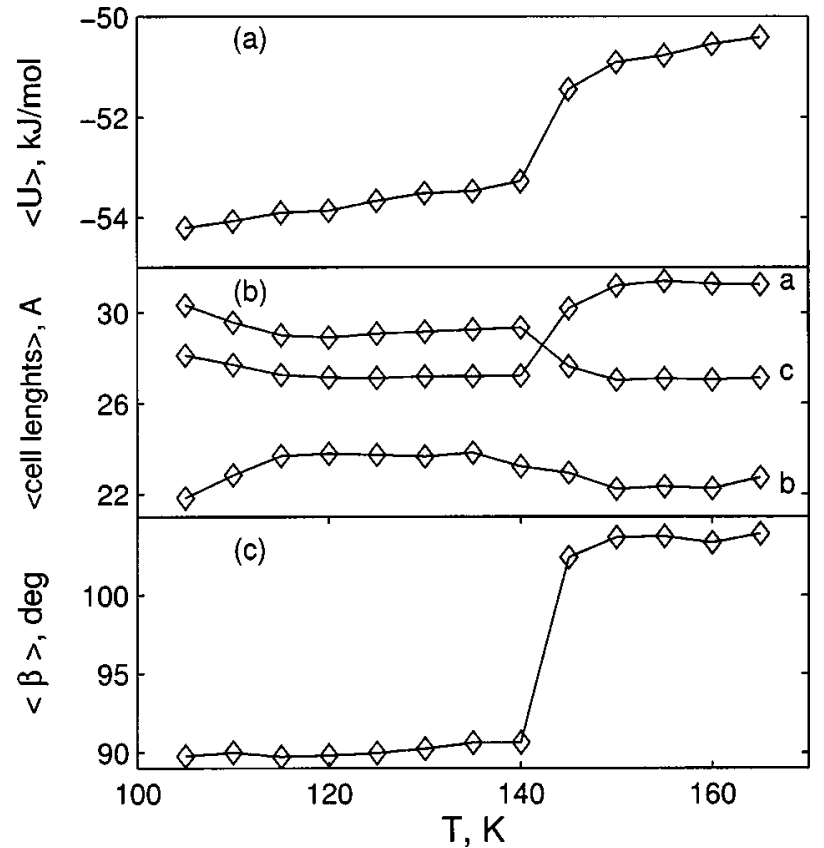

FIG. 11. Variation of the (a) intermolecular interaction energy $U$ [see Eq. (4)] and (b) the simulation cell parameters $a, b$, and $c$ as well as (c) the angle $\beta$ at various temperatures in steps of $5 \mathrm{~K}$ during the heating run. The transition is sharp and suggests a first-order phase transformation between 140 and $145 \mathrm{~K}$. The actual points from run IIIa are shown by $\diamond$.

transition has been successfully simulated in a polyatomic system in which such a large difference in magnitude of one of the cell parameters exists between the starting and the final phases and the transformation being investigated is not an order-disorder transition; obviously, simple doubling of the $\mathbf{a}, \mathbf{b}$ or $\mathbf{c}$ does not belong to this class. However, the large change in $\beta$ is nontrivial. The successful simulation of $\beta$ - to $\alpha$-phase of toluene suggests that the variable shape MC and presumably MD simulations can be employed in realistic studies of crystal structure transformations of at least polyatomic systems.

\section{D. $\alpha$ - to $\beta$-phase structural transformation}

The $\alpha$-toluene at $165 \mathrm{~K}$ was cooled to $105 \mathrm{~K}$ abruptly as well as gradually to see if it transformed back to the $\beta$-phase. Even after repeated attempts, no such transformation was observed; the $\alpha$-phase persisted even at low temperature with little or no change in the structure as evidenced by the various radial distribution functions and orientational correlation functions (not shown). Later we found that even laboratory experiments to transform the monoclinic $\alpha$-phase to the

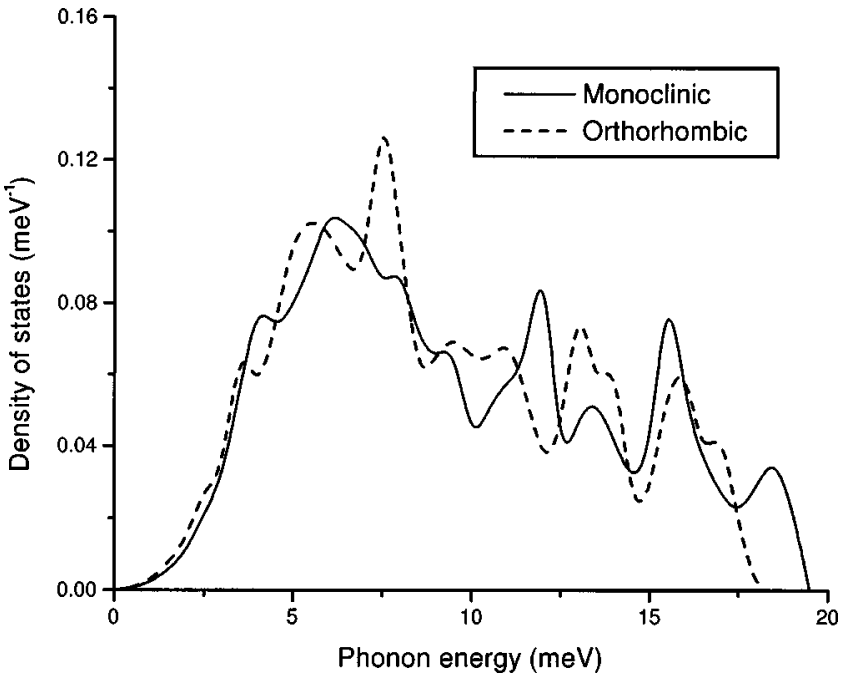

FIG. 12. Density of states within the harmonic approximation using the $0 \mathrm{~K}$ structure.

$\beta$-phase by cooling were unsuccessful and that the $\beta$ - to $\alpha$-phase transformation is indeed irreversible. ${ }^{15}$

\section{E. Phonon spectra}

The phonon density of states (DOS) was calculated within the harmonic approximation and the external mode formalism ${ }^{19,23-26}$ by diagonalization of the dynamical matrices at 128 wave vectors in the Brillouin zone. As there are a total of 48 external mode phonon frequencies at each wave vector corresponding to the 8 molecules in the unit cell in both monoclinic and the orthorhombic structures, we sampled $128 \times 48$ phonon frequencies to calculate the density of states in each structure. Figure 12 shows the phonon DOS spectra for the two phases. Both phases are dynamically stable because the eigenvalues for the $\beta$-phase as well as the $\alpha$-phase are positive at $T=0$ within the harmonic approximation. The spectra are shifted to slightly lower energies for the orthorhombic phase and this leads to a higher vibrational entropy for this phase. Table III lists the vibrational mean square amplitudes along three directions in the monoclinic and orthorhombic phases obtained from within the harmonic approximation and the Monte Carlo simulation. At $T=0$, orthorhombic phase is the stable phase, within the harmonic approximation. The results from the Monte Carlo simulation for the $\beta$ - and the $\alpha$-phase were obtained at $105 \mathrm{~K}$ and 165 $\mathrm{K}$, respectively. Figure 13 shows the center-of-mass trajectory of toluene over a period of $300 \mathrm{MC}$ steps, for $\beta$ - and $\alpha$-phases. The change in free energy between the $\beta$ - and

TABLE III. Vibrational mean square amplitudes in the orthorhombic and monoclinic phases obtained from Monte Carlo and within the harmonic approximation.

\begin{tabular}{lcccccccc}
\hline \hline & \multicolumn{3}{c}{$\beta$-phase $(105 \mathrm{~K})$} & & \multicolumn{3}{c}{$\alpha$-phase $(165 \mathrm{~K})$} \\
\cline { 2 - 3 } \cline { 7 - 8 } & Along $x, \AA^{2}$ & Along $y, \AA^{2}$ & Along $z, \AA^{2}$ & & Along $x, \AA^{2}$ & Along $y, \AA^{2}$ & Along $z, \AA^{2}$ \\
\hline Monte Carlo & 0.04508 & 0.02654 & 0.02474 & & 0.06352 & 0.05015 & 0.05337 \\
Lattice dynamics $^{\mathrm{a}}$ & 0.01915 & 0.01575 & 0.01381 & & 0.01451 & 0.01521 & 0.01680 \\
\hline \hline
\end{tabular}

${ }^{a}$ These results are at $T=100 \mathrm{~K}$ for both $\beta$ - and $\alpha$-phases 


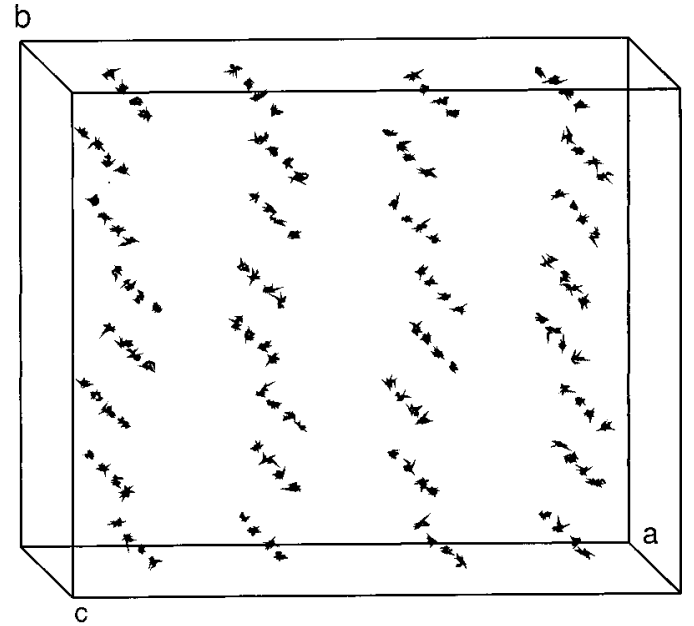

(a)

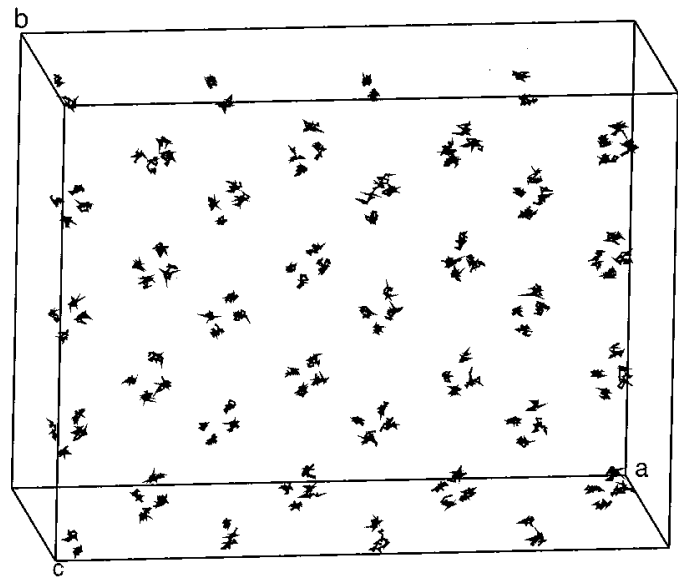

(b)

FIG. 13. Center-of-mass trajectories of toluene over a period of $300 \mathrm{MC}$ steps for (a) $\beta$-phase at $105 \mathrm{~K}$ and (a) $\alpha$-phase at $165 \mathrm{~K}$. Only one in ten steps have been plotted.

$\alpha$-phases, $\Delta G=16 \mathrm{meV} /$ molecule or $1.54 \mathrm{~kJ} / \mathrm{mol}$ from the lattice dynamics calculation. $\beta \rightarrow \alpha$ phase change occurs at $145 \mathrm{~K}$ in the Monte Carlo perhaps due to anharmonicity. The reverse transition does not occur probably due to the kinetics.

\section{F. Liquid toluene}

Toluene is known to melt at $178 \mathrm{~K}$ at atmospheric pressure. ${ }^{27}$ In order to the study the structure of liquid toluene, we first raised the temperature of $\alpha$-toluene from $165 \mathrm{~K}$ to $500 \mathrm{~K}$. The system was retained at $500 \mathrm{~K}$ for $3000 \mathrm{MC}$ steps and subsequently cooled down to $240 \mathrm{~K}$. The system was equilibrated for $1000 \mathrm{MC}$ steps at $240 \mathrm{~K}$ and properties averaged over $2000 \mathrm{MC}$ steps. The volume is somewhat higher (162.4 $\AA^{3} /$ molecule) which corresponds to a density of $0.9477 \mathrm{~g} / \mathrm{cm}^{3}$. The density at $293.15 \mathrm{~K}$ is $0.8669 \mathrm{~g} / \mathrm{cm}^{3}{ }^{27}$ The coefficient of thermal expansion ${ }^{27}$ for toluene has been reported to be $\beta=1.0999 \times 10^{-3} \mathrm{~K}^{-1}$ from which one obtains a value of $0.9547 \mathrm{~g} / \mathrm{cm}^{3}$ (or $161.19 \AA^{3} /$ molecule) for the density at $240 \mathrm{~K}$. This compares well with the computed value. The error in the computed density is $0.72 \%$.

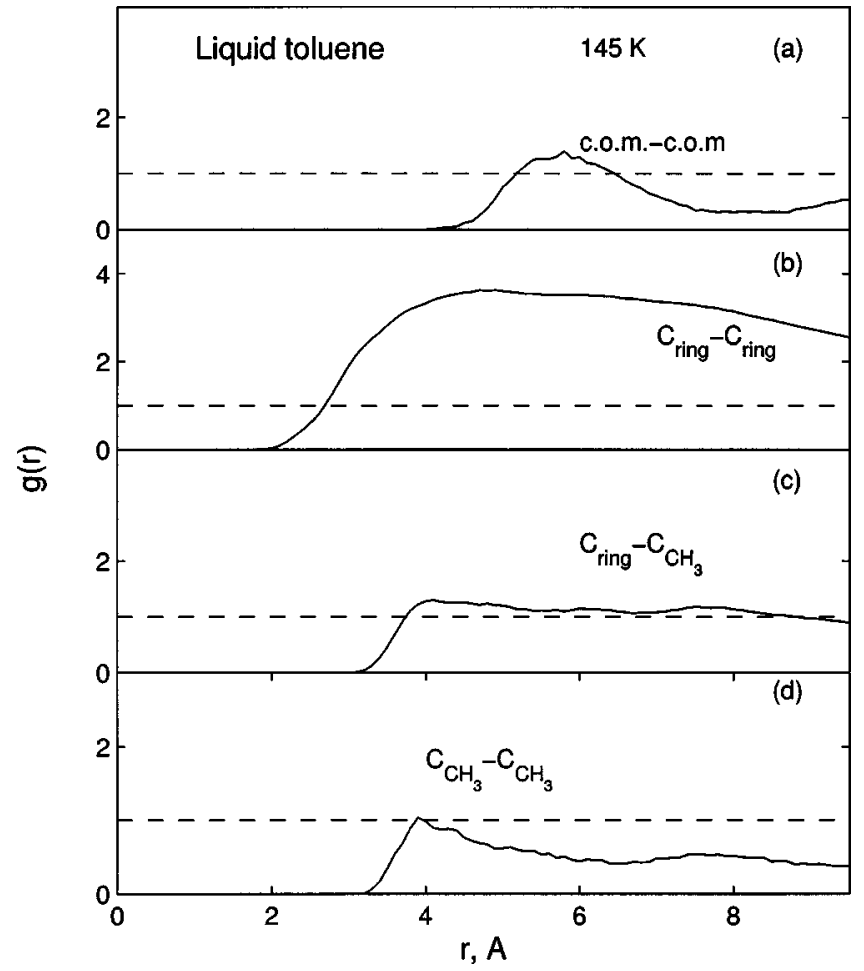

FIG. 14. The radial distribution functions c.o.m.-c.o.m., $\mathrm{C}_{\text {ring }}-\mathrm{C}_{\text {ring }}$ $\mathrm{C}_{\text {ring }}-\mathrm{C}_{\mathrm{CH}_{3}}$, and $\mathrm{C}_{\mathrm{CH}_{3}}-\mathrm{C}_{\mathrm{CH}_{3}}$ of liquid toluene at $240 \mathrm{~K}$.

The average intermolecular potential energy is found to be $-41.25 \mathrm{~kJ} / \mathrm{mol}$. The PV term contributes a small amount $(0.009 \mathrm{~kJ} / \mathrm{mol})$. The enthalpy at $240 \mathrm{~K}$ is therefore around $41.24 \mathrm{~kJ} / \mathrm{mol}$. Experimentally, the heat of vaporization under standard temperature and pressure conditions is 39.20 $\mathrm{kJ} / \mathrm{mol} .{ }^{27}$ The boiling point of toluene is $383.7 \mathrm{~K}$ which is much higher than $240 \mathrm{~K}$ at which we have carried out the simulation.

The rdfs (see Fig. 14) exhibit typical characteristics of any liquid. The orientational correlation functions $\phi_{202}(r)$ and $\phi_{220}(r)$ (see Fig. 15) are both initially positive (i.e., at small $r$ ) suggesting a stacked configuration. It appears that the presence of methyl group lends stability to the stacked configuration in spite of the presence of the quadrupolar interaction. Thus the nearest neighbor structure of the liquid

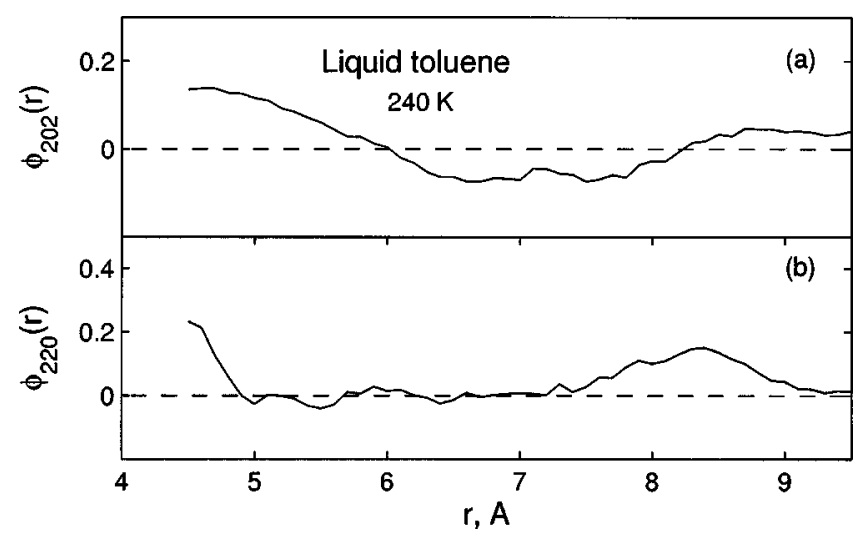

FIG. 15. The orientational correlation functions $\phi_{202}(r)$ and $\phi_{220}(r)$ of liquid toluene at $240 \mathrm{~K}$. 
phases of toluene and benzene are quite different; in benzene liquid, nearest neighbors do not assume a stacked configuration. However the number of pairs with stacked configurations in toluene is very small as is seen from the c.o.m.c.o.m. $\mathrm{g}(r)$. The $\mathrm{g}(r)$ peaks at $6 \AA$ and at this distance the preferred arrangement appears to be $\mathrm{V}$-shaped in which the angle between the rings is around $45^{\circ}$.

\section{CONCLUSIONS}

In conclusion, the parameter set II of the potential proposed by Williams and Starr does reproduce the $\alpha$-phase well. The $\beta$-phase is not reproduced as well. There are several discrepancies between the nearest neighbor structure suggested by $\mathrm{x}$-ray and the simulation. The c.o.m-c.o.m $\mathrm{g}(r)$ of the $\beta$-phase and the orientational correlation function of the $\alpha$-, as well as the $\beta$-phase exhibit deviations from the experimental structure. These functions provide a much more rigorous test of the Williams and Starr potential. One of the general discrepancies of the Williams potential appears to be an overestimate of the heat of vaporization. Earlier study on benzene also found a similar trend. ${ }^{14}$ Lattice dynamical calculations suggest that within harmonic approximation the transition does not occur.

It is unlikely that the parameter set I given by Williams and Starr will do as well as set II since the former does not have any electrostatic interactions. The potential of Williams and Starr reproduces the transition from the $\beta$ - to $\alpha$-phase successfully. In the simulation the reverse transition does not take place. This is precisely what is observed experimentally. This appears to be the first successful simulation of a phase transformation in a polyatomic system apart from the usual order-disorder type of transformations. This study suggests that the study of the condensed phases of aromatic solids can be carried out successfully using the variable shape simulation methods and the potentials existing in the literature. The present study suggests some refinement in the existing potential. It also suggests that the six degrees of freedom for the simulation cell proposed earlier by Yashonath and Rao does not in any way restrict or inhibit the transformation. This suggests that while such a restriction prevents the rotation of the simulation cell, it does not in any way inhibit the system from transforming to any other structure that is consistent with the intermolecular potential.

\section{ACKNOWLEDGMENTS}

The authors thank the Council of Scientific and Industrial Research, New Delhi for partial financial support in carrying out this work. One of us (A.V.A.K.) wishes to thank University Grants Commission, New Delhi for the award of a senior research fellowship. Contribution No. 1517 from the Solid State and Structural Chemistry Unit.

${ }^{1}$ A. I. Kitaigorodskii, Molecular Crystals and Molecules (Academic, New York, 1973)

${ }^{2}$ A. Gavezzotti, Acc. Chem. Res. 27, 309 (1994).

${ }^{3}$ G. R. Desiraju, Crystal Engineering (Elsevier, Amsterdam, 1989).

${ }^{4}$ G. R. Desiraju and A. Gavezzotti, Acta Crystallogr., Sect. B: Struct. Sci. 45, 473 (1989).

${ }^{5}$ M. Parrinello and A. Rahman, Phys. Rev. Lett. 45, 1196 (1980).

${ }^{6}$ S. Yashonath and C. N. R. Rao, Mol. Phys. 54, 245 (1985).

${ }^{7}$ S. Nose and M. L. Klein, Phys. Rev. Lett. 50, 1207 (1983).

${ }^{8}$ M. Parrinello, A. Rahman, and P. Vasishta, Phys. Rev. Lett. 50, 1073 (1983).

${ }^{9}$ S. Nose and M. L. Klein, Mol. Phys. 50, 1055 (1983); J. Chem. Phys. 78, 6928 (1983)

${ }^{10}$ S. Yashonath and C. N. R. Rao, Chem. Phys. Lett. 119, 22 (1985).

${ }^{11}$ E. Nuesy, S. Nose, and M. L. Klein, Mol. Phys. 52, 269 (1984).

${ }^{12}$ S. Yashonath and C. N. R. Rao, J. Phys. Chem. 90, 2552 (1986).

${ }^{13}$ A. Chakrabarti, S. Yashonath, and C. N. R. Rao, Mol. Phys. 84, 49 (1995).

${ }^{14}$ S. Yashonath, S. L. Price, and I. R. McDonald, Mol. Phys. 64, 361 (1988).

${ }^{15}$ D. Andre, R. Fourme, J. Bruneaux-Poulle, and L. Bosio, J. Mol. Struct. 81, 253 (1982).

${ }^{16}$ M. Anderson, L. Bosio, J. Bruneaus-Poulle, and R. Fourme, J. Chim. Phys. 74, 68 (1977).

${ }^{17}$ D. E. Williams and T. L. Starr, Comput. Chem. 1, 173 (1977).

${ }^{18}$ D. E. Williams and S. R. Cox, Acta Crystallogr. B 40, 404 (1984).

${ }^{19}$ S. L. Chaplot, Phys. Rev. B 36, 8471 (1987).

${ }^{20}$ M. Metropolis, A. Rosenbluth, M. N. Rosenbluth, A. N. Teller, and E. Teller, J. Chem. Phys. 21, 1087 (1953).

${ }^{21}$ O. Steinhauser, Chem. Phys. 73, 155 (1982).

${ }^{22}$ M. Parrinello and A. Rahman, in Melting, Localization, and Chaos, edited by R. K. Kalia and P. Vashishta (Elsevier, Amsterdam, 1982).

${ }^{23}$ G. Venkataraman and V. C. Sahni, Rev. Mod. Phys. 42, 409 (1970).

${ }^{24}$ K. R. Rao, S. L. Chaplot, P. K. Iyengar, A. H. Venkatesh, and P. R. Vijayaraghavan, Pramana 11, 251 (1978)

${ }^{25}$ S. L. Chaplot, External Report, BARC, 972, 1978.

${ }^{26}$ G. S. Pawley, in Methods of Experimental Physics, edited by K. Scold and D. Price (Academic, New York, 1986), Vol. A23, p. 441.

${ }^{27}$ CRC Handbook of Chemistry and Physics, edited by R. C. Weast (CRC Press, Cleveland, 1976-77). 